\title{
CUMULATIVE AUTHOR INDEX
}

(Volume 1)

\author{
Akemann, W. 557 \\ Al-Achkar, M. 665 \\ Allison, W. 509 \\ Andersson, S. 187 \\ Andrews, A. B. 565 \\ Apai, G. 635 \\ Árko, A. J. 565 \\ Armstrong, S. R. 485 \\ Avery, A. R. 621 \\ Bailey, P. 597 \\ Baker, S. H. 525 \\ Balakrishnan, G. 545 \\ Barbier, L. 75 \\ Barnes, C. 569 \\ Barrett, S. D. 625,649 \\ Bartolucci, F. 561 \\ Baschenko, O. A. 577 \\ Bass, J. M. 481 \\ Beamson, G. 545 \\ Bellman, A. 67 \\ Berger, M. G. 585 \\ Binns, C. R. 525 \\ Blomfield, C. J. 669 \\ Blyth, R. I. R. $565,649,701$ \\ Bohnen, K. P. 97 \\ Boronin, A. I. 577 \\ Bortolani, V. 67 \\ Bowker, M. 549, 569 \\ Bridges, A. S. 573 \\ Bukhtiyarov, V. I. 577 \\ Bullman, H. 509 \\ Burke, K. 133, 175 \\ Burstein, E. 47 \\ Bush, T. L. 581 \\ Butler, D. 693 \\ Canfield, P. C. 565 \\ Cavanagh, M. 585 \\ Chang, T.-C. 197 \\ Chen, C.-L. 197 \\ Chen, Y. 97 \\ Chow, J. C. L. 37 \\ Cini, M. 443 \\ Cole, R. J. 469 \\ Collins, I. 685 \\ Condon, N. G. 705 \\ Cooper, B. H. 175 \\ Cooper, E. 465
}

Cottam, M. G. 23, 239

Cottrell, C. 501

Crapper, M. D. 641

Cvetko, D. 67

D'Addato, S. 597, 601

Darling, G. R. 115

Dastoor, P. C. 509

Dederichs, P. H. 477

Delong, L. 565

Derbyshire, H. S. 525

Devine, P. 669

Dharmadasa, I. M. 669

Dhesi, S. S. 625,649

Di Nardo, S. 589

Diehl, R. D. 529

Dirubio, C. A. 175

Dixon-Warren, St. J. 593

Dobrodey, N. V. 273

Duchinsky, V. G. 285

Dwyer, J. 697

Ellis, J. 509

Evans, D. A. 597

Fasel, R. 229

Favorski, I. A. 239

Fawcette, P. N. 485

Finetti, P. 601

Fisk, Z. 565

Floreano, L. 67

Flowers, M. C. 505

Franchini, A. 67

Francis, S. M. 569

Frederick, B. G. 635

Fung, P. C. W. 37

Gardner, P. 661, 673

Gates, G. 605

Gillan, M. J. 491

Gleeson, M. 569

Goodman, D. W. 449

Goodstein, D. M. 175

Gravil, P. A. 611

Greef, R. 573

Gregory, G. C. 669

Greig, D. 545

Gumhalter, B. 133

Guo, Q. 539
Håkansson, L. 701

Harris, S. 615

Hayden, B. E. 693

Hayward, D. O. 581

Heinemann, M. 429

Heinz, K. 319

Ho, K. M. 97

Hodgson, A. 501, 693

Holloway, S. 115, 605, 611, 615

Holmes, D. M. 621

Holst, B. 509

Hoogers, G. 553

Hopster, H. 89

Horio, Y. 1

Howes, P. B. 631

Huang, H. 221, 303

Ichimiya, A. 1

Ignatiev, A. 253, 261

Inglesfield, J. E. 415, 685

Inkson, J. C. 473

Iwatsuki, M. 1

Jacobi, K. 221

James, M. A. 631

Jenkins, S. J. 473

Jona, F. 15,411

Jonathan, N. B. H. 505, 573

Jones, R. G. 535

Jones, T. S. 513, 581, 621

Joyce, B. A. 485,621

Joyce, J. J. 565

Kadodwala, M. 535

Keen, A. M. 525

King, D. A. 435, 517, 553, 593, $661,673,689$

Kleyn, A. W. 157

Kohn, W. 129

Kono, S. 359

Kuyanov, I. A. 273

Kuznetsova, T. 239

Lahiase, U. G. L. 565

Lambert, R. M. 655

Lang, P. 477

Langreth, D. C. 133

Lee, M. B. 635

Leibsle, F. M. 513, 625, 705

Levi, A. C. 109 
Li, H. 303

Liang, W. Y. 545

Liddiard, A. J. 641

Liebsch, A. 521

Lifshits, V. G. 285

Lin, C. T. 545

Lindsay, R. 705

Lo, R.-L. 197

Lozzi, L. 589

Manassidis, I. 491

Mapledoram, L. D. 517

Marcus, P. M. 15, 411

Matthai, C. C. 481

Matthew, J. A. D. 545

Matthews, G. W. 669

McConville, C. F. 513

McGilp, J. F. 585

McGrath, R. 529

Mills, D. L. 47

Morgante, A. 67

Morris, A. 505, 573

Morris, S. J. 481

Mozley, S. N. 525

Münder, H. 585

Munro, S. 645

Murray, S. J. 529

Myler, U. 221

Nagayoshi, H. 369

Neave, J. H. 485

Nekovee, M. 415

Neugebauer, J. 213

Newton, M. 569

Nicklin, C. L. 631

Nogami, J. 395

Nomura, H. 1

Norris, C. 525, 631

Osterwalder, J. 229

Otto, A. 557, 561

Papageorgopoulos, D. C. 553

Parker, G. J. 573

Parker, T. M. 705

Passacantando, M. 589

Passerone, D. 109

Pasteur, A. T. 593
Patchett, A. J. 649

Patel, R. B. 641

Peloi, M. 67

Pemble, M. E. 485

Persson, M. 187

Petukhov, A. V. 521

Picozzi, P. 589

Poulston, S. 655

Power, J. R. 585

Price, N. J. 701

Raval, R. 465, 645

Reichmuth, A. 509

Reinhardt, F. 481

Richardson, N. V. 513

Richter, W. 481

Rieder, K. H. 51

Rieley, H. 701

Robinson, A. W. $601,625,701$

Rodach, T. 97

Rojdestvenski, I. V. 239

Rose, K. C. 481

Rumberg, J. 481

Ryzhkov, S. V. 285

Salanon, B. 75

Salman, A. 23

Sanders, H. E. 661

Sands, D. 669

Santoro, G. 67

Santucci, S. 589

Sato, T. 1

Scheffler, M. 213

Schlapbach, L. 229

Scott, C. G. 665,669

Shen, T.-H. 545

Sim, W. S. 673

Sprösser, J. 75

Srivastava, G. 677

Srivastava, G. P. $473,495,681$

Stampfl, C. 213

Stepanyuk, V. S. 477

Sterratt, D. 539

Stuck, A. 229

Stuckless, J. T. 689

Sueyoshi, T. 1
Taylor, A. G. 485

Thornton, G. 601, 705

Thornton, J. M. C. 597

Tikhov, M. 655

Tobin, J. G. 297

Tommasini, F. 67

Tong, S. Y. 97, 221, 253, 261, 303

Tsong, T. T. 197

Turner, A. R. 485

Turner, T. S. 631

Turton, S. 535

Umerski, A. 495, 681

Van Hove, M. A. 9

van Hoof, J. 685

van Silfhout, R. G. 631

Vorontsov-Velyaminov, P. N. 239

Waddill, G. D. 297

Walker, C. G. H. 545

Wander, A. 517

Wartnaby, C. E. 689

Wedler, H. 319

Wei, C. M. 335

Weightman, P. 469

Westwood, D. I. 481

White, R. G. 701

Wight, A. 501, 693

Wildberger, K. 477

Williams, E. M. 539

Williams, R. H. 481

Woodruff, D. P. 457

Woolf, D. A. 481

Worthy, G. 501, 693

Wright, S. 505

Yousaf, M. 669

Zahn, D. R. T. 421

Zambelli, T. 67

Zavodinsky, V. G. 273

Zeller, R. 477

Zhang, J. 485

Zhao, T. C. 253, 261

Zholobenko, V. L. 697

Ziegelman, L. I. 273

Zotov, A. V. 285 
Apel, R. 153

Bayer, P. 89

Bousetta, A. 171

Bukaluk, A. 191

Cabrera, A. L. 159

Courths, R. 147

Crommie, M. F. 127

De Crescenzi, M. 255

Delmon, B. 25

Demangeat, C. 203

Dreyssé, H. 203

Eigler, D. M. 127

Erbudak, M. 141

Farías, D. 153

Garrido-Molina, W. H. 159

Goodman, D. W. 9

Heinz, K. 89

Heise, R. 147

Heller, E. J. 127

Hennig, D. 197

Hergert, W. 203

Himpsel, F. J. 81

Hitchcock, A. P. 43

Hochstrasser, M. 141
Hsu, C. C. 171

$\mathrm{Hu}, \mathrm{C} .245$

Johansson, L. I. 225

Jona, F. 183

Kim, S. K. 183

Kuyanov, I. A. 219

Lad, R. J. 109

Li, Y. S. 165

Lozzi, L. 255

Lutz, C. P. 127

Marcus, P. M. 183

Methfessel, M. 197

Minoda, H. 1

Mitchell, K. A. R. 165

Müller, S. 89

Nicholson, D. M. C. 71

Passacantando, M. 255

Pescia, D. 141

Picozzi, P. 255

Rehr, J. J. 63

Ren, D. G. 177

Rennert, P. 203

Rieder, K. H. 153
Santucci, S. 255

Scheffler, M. 197

Shelton, W. A. 71

Sheu, B.-R. 183

Stocks, G. M. 71

Strongin, D. R. 183

Szotek, Z. 71

Tanishiro, Y. 1

Temmerman, W. M. 71

Tromp, R. M. 103

Tyliszczak, T. 43

Volkmann, U. G. 159

Wang, C. P. 183

Wang, Y. 71

Wang, Y. 171

Wetli, E. 141

Wong, P. C. 165

Yagi, K. 1

Yamamoto, N. 1

Yang, W. S. 245

Zavodinsky, V. G. 219

Zhao, R. G. 245

Zhou, M. Y. 165 